Notes \& Tips

\title{
Two complementary fluorimetric assays for the determination of aminoquinoline binding and uptake by human erythrocytes in vitro
}

\author{
Nicoletta Basilico, Lucia Cortelezzi, Chiara Serpellini, Donatella Taramelli, Fausta Omodeo Salè * \\ Institute of General Physiology and Biochemistry “G. Esposito”, Department of Public Health-Microbiology-Virology, Faculty of Pharmacy, University of Milan, 20134 Milan, Italy
}

\section{A R T I C L E I N F O}

\section{Article history:}

Received 14 August 2008

Available online 13 November 2008

\begin{abstract}
A B S T R A C T
We provide two simple low-cost and low-tech procedures to measure with good precision and accuracy the binding and internalization into human erythrocytes of chloroquine and other aminoquinolines. The methods are based on the high fluorescence of the quinoline ring and are complementary. Method A evaluates residual drugs in the supernatants of treated erythrocytes, whereas method B quantifies the total uptake by whole cells and the fraction bound to the membranes. Drug uptake is dose dependent and related to the number of erythrocytes. These assays could be useful when studying the cell interaction of quinoline-type compounds not available in the radioactive form.
\end{abstract}

(c) 2008 Elsevier Inc. All rights reserved.
Chloroquine $(\mathbf{C Q})^{1}$ has been one of the most successful antimalarial drugs due to its specificity, safety, and stability, but it is now largely ineffective in most malaria endemic areas because of the emergence of Plasmodium falciparum $(P f)$ resistance. Artemisin-based combination therapy is currently recommended as first-line treatment for uncomplicated malaria, but there is a continuous necessity of new drugs or new drug combinations to increase efficacy and delay the onset of resistance $[1,2]$.

We recently synthesized new quinolizidinyl and quinolizidinylalkyl derivatives of 4-aminoquinolines in which a terminal bulky bicyclic basic moiety was introduced to prevent the metabolic oxidation that limits the usefulness of quinoline compounds. Leads that are highly effective in vitro against several drug-resistant strains of $P f$ and in vivo in the murine Plasmodium berghei model were obtained [3]. Two of these compounds, named AM1 and AP4b, were selected for further characterization (Fig. 1). Preliminary pharmacokinetic data indicate that more than $60 \%$ of compounds are bound to the corpuscular blood fraction. This is consistent with what has been described previously for CQ [4].

Since the early 1970s, the capacity of red blood cells (RBC) uninfected or infected by different strains of $P f$ to bind $\mathbf{C Q}$ or other aminoquinoline antimalarial drugs has been evaluated using ${ }^{3} \mathrm{H}$ - or ${ }^{14} \mathrm{C}$-labeled compounds. The uptake of the drug by RBC was quantified by counting the radioactivity that is associated with the cell pellet or that disappeared from the incubation medium after centrifugation [5-7]. To avoid the laborious synthesis and risky use of radiolabeled compounds, we developed two simple, low-cost,

\footnotetext{
* Corresponding author. Fax: +390250315775.

E-mail address: fausta.omodeosale@unimi.it (F. Omodeo Salè).

${ }^{1}$ Abbreviations used: CQ chloroquine; Pf, Plasmodium falciparum; RBC, red blood cells; PBS, phosphate-buffered saline; IF, intensity of fluorescence; Hb, hemoglobin; Htc, hematocrit; FP, ferriprotoporphyrin IX.
}

and reproducible methods based on the high fluorescence of the quinoline ring to evaluate binding/internalization of the quinolines to human RBC.

Method A measures the residual fluorescence in the supernatants of drug-treated RBC, whereas method B was optimized to differentiate and quantify the amount of compound bound to RBC membranes or internalized by RBC. The experiments were done using fresh RBC from healthy donors. Cells were washed three times with $5 \mathrm{mM}$ cold phosphate-buffered saline (PBS)-glucose and incubated with the drugs at $37^{\circ} \mathrm{C}$ in the optimized conditions described below.

In preliminary experiments, we determined the fluorescence spectra of the quinolines under study. For each compound, the excitation wavelengths $(\mathbf{C Q}=258 \mathrm{~nm}, \mathbf{A M 1}=255 \mathrm{~nm}, \mathbf{A P 4 b}=$ $253 \mathrm{~nm}$ ) corresponding to the maximum emission at $380 \mathrm{~nm}$ were chosen. Specific excitation wavelengths must be properly selected for each quinoline derivative.

In addition, because quinolines are weak bases, the intensity of fluorescence (IF) is strongly dependent on $\mathrm{pH}$, with the highest IF being in highly basic media $(\mathrm{pH}>12.0)$ in the deprotonated form. For example, at acidic pH, CQ IF is negligible, whereas at neutral $\mathrm{pH}$ (PBS), IF is approximately $45 \%$ compared with that measured at $\mathrm{pH}>12.0$ (not shown). Two factors could contribute to the lower fluorescence at neutral $\mathrm{pH}$ : (i) a reduced electronic transition due to the partially protonated form of the drug and (ii) self-quenching due to $\pi-\pi$ interaction among the quinoline rings. A linear relationship between $\mathbf{C Q}$ concentration and IF was observed between 0 and $2 \mu \mathrm{M}$ with a detection limit at $0.15 \mu \mathrm{M}$. Concentrations of CQ higher than $2 \mu \mathrm{M}$ were not linearly related to IF (not shown). AM1 and AP4b behaved similarly. Variations in temperature do not interfere with IF.

For method A, preliminary experiments were performed to standardize the method and minimize the interference of hemo- 
<smiles>CCN(CC)CCCC(C)Nc1ccnc2cc(Cl)ccc12</smiles>

Chloroquine<smiles>CNc1ccnc2cc(Cl)ccc12</smiles>

AM1<smiles>Clc1ccc2c(NC3CCCN4CCCCC34)ccnc2c1</smiles>

AP4b

Fig. 1. Molecular structures of the compounds used in this study.

globin $(\mathrm{Hb})$ on the IF of the tested compounds. To avoid an inner filter effect leading to misinterpretation of the data, the measurements were performed in very diluted samples whose absorbance at the excitation wavelength was less than 0.07. During the assay, trace amounts of $\mathrm{Hb}$ may be released by RBC depending on hematocrit (Htc). Hb fluorescence from control RBC was evaluated and subtracted from all samples. In addition, free $\mathrm{Hb}$ may cause quenching of IF of the drugs. For this reason, the emission spectrum of the drug in the presence of the supernatant of RBC was preferred as a control instead of the spectrum of the drug alone. The quenching due to the presence of free $\mathrm{Hb}$ is measured by the difference of IF between the two spectra.

The assay was performed by incubating RBC at $1 \%$ to $4 \% \mathrm{Htc}$ in PBS for different lengths of time (10-180 min) in the presence of drug concentrations ranging from 1 to $0.25 \mu \mathrm{M}$ in 24-well plates at a final volume of $2 \mathrm{ml}$. The incubation was performed at room temperature or $37^{\circ} \mathrm{C}$ in an incubator without $\mathrm{CO}_{2}$ to avoid the acidification of the medium and the decrease of IF. Plates were then centrifuged at $600 \mathrm{~g}$ for $10 \mathrm{~min}$, and supernatants were recovered for fluorimetric analysis using a PerkinElmer LS50 fluorimeter. Quartz cuvettes were always used for the fluorescence measurements. The areas of the peaks were calculated by FLWinLab software.

Binding of the different quinolines to RBC was very rapid (plateau after $10 \mathrm{~min}$ ) and dependent on the concentration of RBC. The experimental conditions allowing a rapid and sensitive analysis, free of significant quenching and/or interferences, were set as follows: $2 \% \mathrm{Htc}, 10 \mathrm{~min}$ incubation, and $1 \mu \mathrm{M}$ drugs at $37{ }^{\circ} \mathrm{C}$ or room temperature (binding was not significantly different at the two temperatures). The method was optimized for cuvette measurement, but it could be easily adapted to microtiter wells and a fluorescent plate reader. Fig. 2A reports the emission spectra from a representative experiment in which binding of AM1 to RBC was evaluated. The continuous top line (a) is the fluorescence of AM1 at $1 \mu \mathrm{M}$. The dashed line (b) represents the fluorescence of AM1 measured in the presence of RBC supernatant; it shows the quenching of AM1 by free $\mathrm{Hb}$ and is used as a reference spectrum instead of the top line (a). The decreased emission of AM1 in the presence of RBC, which indicates binding of AM1 to RBC, is depicted by the dotted line $(\mathrm{c})$. The bottom line (d) represents the baseline fluorescence of free $\mathrm{Hb}$ in the supernatants from control RBC.

Therefore, for each experiment, the percentage binding of each drug to RBC can be calculated using the following equation:

\%binding $=(c-d) /(b-d) \times 100$,

where the letters represent the areas under the curve of the corresponding spectra. In the experiment of Fig. 2A, 20\% of AM1 fluorescence disappeared from the supernatant of treated RBC. This corresponds to approximately $400 \mathrm{pmol}$ of AM1 bound/internalized by RBC at $2 \%$ Htc. Because method A gives an indirect estimate of the amount of drug bound to RBC, method B was developed to pro- vide a precise quantification and the cellular distribution of the drugs.

For method B, the first set of tests was conducted to find the best experimental conditions for a quantitative extraction of CQ from the RBC suspension, minimizing interferences due to the presence of ferriprotoporphyrin IX (FP), a by-product of Hb degradation. The experiments were conducted by extracting mixtures made of different amounts of CQ $(0.5-25 \mathrm{nmol})$ and RBC (50$150 \mu \mathrm{l}$ of packed RBC corresponding to $0.5-1.5 \times 10^{9}$ cells). Samples were subjected to an organic extraction in acidic conditions by $1 \mathrm{ml}$ of $\mathrm{CHCl}_{3} / 1 \mathrm{~N} \mathrm{HCl}(0.8: 1, \mathrm{v} / \mathrm{v})$. The suspension was mixed vigorously and centrifuged at maximum speed for $10 \mathrm{~min}$. By this protocol, CQ is solubilized in the protonated form in the aqueous
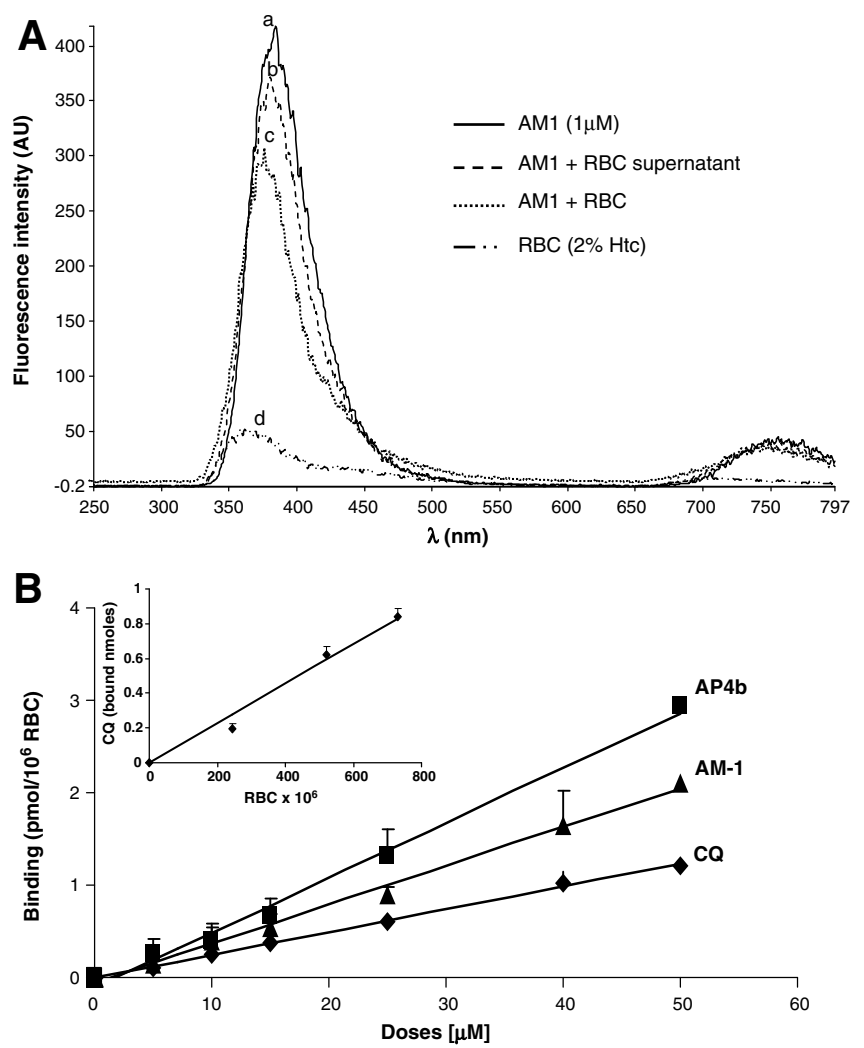

Fig. 2. (A) Method A: representative experiment showing the decrease of AM1 fluorescence when incubated with human RBC. Shown are fluorescence emission spectra of $1 \mu \mathrm{M}$ AM1 (excitation at $255 \mathrm{~nm}$ ) alone (a: continuous line), in the presence of RBC supernatant ( $2 \% \mathrm{Htc}$ in $2 \mathrm{ml}$ final volume) (b: dashed line), or in the presence of RBC (c: dotted line). The bottom line (d) represents the baseline fluorescence of free $\mathrm{Hb}$ in the supernatants from control RBC. AU, arbitrary units. (B) Method B: dose-dependent binding of CQ AM1, and AP4b to human RBC. The inset shows uptake of $\mathbf{C Q}$ as function of the number of RBC. Data represent the means of five different experiments run in triplicate. Vertical error bars should be considered as symmetric. 
acid phase, whereas the lipid fraction of $\mathrm{RBC}$ and $\mathrm{Hb}$ are recovered in the organic phase. A proper aliquot of the aqueous acid fraction was diluted to $2 \mathrm{ml}$ with $1 \mathrm{~N} \mathrm{HCl}$ and corrected to basic pH by $245 \mu \mathrm{l}$ of $10 \mathrm{~N} \mathrm{NaOH}$ prior to fluorescence measurements. For each compound, a calibration curve was calculated by extracting increasing concentrations of the drug $(2-25 \mathrm{nmol})$ in a free cell system. Binding of drugs to RBC was quantified by incubating $500 \mu \mathrm{l}$ of RBC at $15 \% \mathrm{Htc}$ in PBS- $5 \mathrm{mM}$ glucose buffer at $37^{\circ} \mathrm{C}$ with different amounts of the drug $(10-50 \mu \mathrm{M})$ and for different times (10-120 min). At the end of incubation, cells were pelleted by centrifugation, washed in PBS-glucose to remove the unbound drug, and processed as described above. Binding was very rapid, reaching $90 \%$ of maximum within 10 min and a plateau after 30 min of incubation (data not shown).

As reported in Fig. 2B, binding of AM1, AP4b, or CQ was dose dependent and linearly related to the amount of drug in the medium. The higher values of binding of AP4b and AM1 can be ascribed to the higher lipophilicity (compared with CQ) of the bicyclic substituent, improving cellular permeation. A linear relation between the number of $\mathrm{RBC}$ and the amount of drug recovered in the acid aqueous phase was observed, with a number of cells equal to or minor of $10^{9}(25-30 \mathrm{mg} \mathrm{Hb})$ (see inset), whereas higher values of Htc did not allow a complete recovery of the drug (data not shown). For this reason, further experiments were performed by incubating $500 \mu \mathrm{l}$ of RBC at $15 \% \mathrm{Htc}$ $\left(750 \times 10^{6} \mathrm{RBC}\right)$.

To distinguish between $\mathbf{C Q}$ internalized and $\mathbf{C Q}$ bound to the membrane, in some experiments at the end of incubation RBC were pelleted, washed in PBS-glucose, and lysed by hypotonic shock as reported previously [8]. Membranes were isolated and washed until free of $\mathrm{Hb}$ and were extracted as described for whole RBC. The amount of CQ entrapped in the membrane was approximately $10 \%$ of the total $\left(0.1 \mathrm{pmol} / 10^{6} \mathrm{RBC}\right.$ vs. $\left.0.98 \mathrm{pmol} / 10^{6} \mathrm{RBC}\right)$. As expected, and in agreement with Bray and coworkers [7], when CQ and RBC were coincubated in the presence of FP $(10 \mu \mathrm{M})$, the drug bound to RBC was more than twice that measured in the absence of FP $\left(2.32 \mathrm{pmol} / 10^{6} \mathrm{RBC}\right)$ and CQ entrapped in the membrane increased from $10 \%$ to approximately $50 \%$ ( $\left.1.3 \mathrm{pmol} / 10^{6} \mathrm{RBC}\right)$.

When methods A and B were compared and the drug concentration was adjusted for the number of RBC, no significant differences were found between the number of picomoles of CQ bound to 106 RBC calculated by method $A$ and that recovered in RBC after extraction by method $\mathrm{B}(0.33 \pm 0.14$ and $0.25 \pm 0.04$, respectively, $n=5)$. Analytical precision and accuracy were evaluated for method B by interassay (10 samples during a 1-week period) and intraassay precision data $(n=10)$ spiked with 10,25 , or $50 \mu \mathrm{M}$ CQ and coefficients of variation were less than $10 \%$.
Preliminary experiments demonstrated that the two methods can be easily applied to Pf-infected RBC. As expected, infected RBC seem to bind more CQ compared with normal RBC. Further investigations are in progress to extend this finding to other quinoline-type compounds.

In summary, we have developed two simple and inexpensive fluorimetric methods to quantify the amount of CQ or related quinolines bound or being internalized by human RBC. The procedure, based on the high fluorescence of the quinoline ring, is rapid, is reproducible, and can afford good precision and accuracy; therefore, it is suitable when comparing new quinolines not available in the radioactive form.

Method A is faster, but it gives an indirect estimate of drug binding to RBC and it could be suggested as initial screening of new compounds. Method B is more time-consuming, but it allows an accurate quantification of the amount of drug bound to RBC membranes or internalized by RBC.

Both methods could provide useful information during the preclinical assessment of the drug likeness of new molecules.

\section{Acknowledgments}

The authors thank A. Sparatore (Istituto di Chimica Farmaceutica e Tossicologica "Pietro Pratesi," University of Milan) for the synthesis of compounds AM1 and AP4b and Diego Monti (ISTM-CNR, Milan, Italy) for helpful discussion. The financial support of the University of Milan (first 2006-2008) and the EU collaborative project ANTIMAL 18834 is acknowledged.

\section{References}

[1] World Health Organization, Antimalarial drug combination therapy ( $\mathrm{CH}$ WHO/ CDS/RBM 35, report of a technical consultation), WHO, Geneva, 2001.

[2] N.J. White, Antimalarial drug resistance, J. Clin. Invest. 113 (2004) 1084-1092.

[3] A. Sparatore, N. Basilico, S. Parapini, S. Romeo, F. Novelli, F. Sparatore, D. Taramelli, 4-Aminoquinoline quinolizidinyl- and quinolizidinylalkyl-derivatives with antimalarial activity, Bioorg. Med. Chem. 13 (2005) 5338-5345.

[4] J. Ducharme, R. Farinotti, Clinical pharmacokinetics and metabolism of chloroquine: focus on recent advancements, Clin. Pharmacokinet. 31 (1996) 257-274.

[5] C.D. Fitch, Chloroquine-resistant Plasmodium falciparum: difference in the handling of ${ }^{14} \mathrm{C}$-amodiaquin and ${ }^{14} \mathrm{C}$-chloroquine, Antimicrob, Agents Chemother. 3 (1973) 545-548.

[6] C.D. Fitch, R. Chevli, Y. Gonzalez, Chloroquine-resistant Plasmodium falciparum: effect of substrate on chloroquine and amodiaquin accumulation, Antimicrob, Agents Chemother. 6 (1974) 757-762.

[7] P.G. Bray, O. Janneh, K.J. Raynes, M. Mungthin, H. Ginsburg, S.A. Ward, Cellular uptake of chloroquine is dependent on binding to ferriprotoporphyrin IX and is independent of NHE activity in Plasmodium falciparum, J. Cell Biol. 145 (1999) 363-376.

[8] F. Omodeo Salè, A. Motti, A. Dondorp, N.J. White, D. Taramelli, Destabilization and subsequent lysis of human erythrocytes induced by Plasmodium falciparum heme products, Eur. J. Haematol. 74 (2005) 324-332. 